\title{
Adecuación del tratamiento inhalado en pacientes diagnosticados de Enfermedad Pulmonar Obstructiva Crónica
}

Gabriel J. Díaz-Grávalosa, Gerardo Palmeiro Fernándezº , María Dolores Valiño-Lópeza, Aída Robles-Castiñeiras ${ }^{c}$, María Jesús Fernández-Silva ${ }^{d}$, Santiago Reinoso-Hermidae, Inmaculada Casado-Górriz ${ }^{\dagger}$

a Centro de Salud Cea (Orense). Servicio Gallego de Salud (SERGAS).

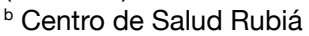
(Orense). SERGAS.

c Centro de Salud Villar de Santos (Orense). SERGAS.

${ }^{a}$ Dirección de Procesos de Soporte. Gerencia de Área Integrada de Orense (Orense). SERGAS.

e Jefe de Estudios. Unidad Docente de Medicina Familiar y Comunitaria. Orense. SERGAS. ${ }^{\dagger}$ Centro de Salud Allariz (Orense). SERGAS.

Correspondencia: Gabriel J. Díaz Grávalos. C/ Feria, 9. C.P. 32130 - Cea (Orense). Teléfono: 988282 401. Correo electrónico: gabriel.diaz. gravalos@sergas.es

Estudio financiado mediante una beca no condicionada por Boehringer Ingelheim España y Pfizer España. Ambas compañías no han influido en modo alguno en la metodología o en la redacción del manuscrito.

Recibido el 27 de septiembre de 2012.

Aceptado para su publicación el 6 de mayo de 2013.

\section{RESUMEN}

Objetivo: Valorar la adecuación a las guías clínicas (GOLD) del tratamiento por vía inhalada de los pacientes diagnosticados de Enfermedad Pulmonar Obstructiva Crónica (EPOC).

Diseño del estudio: Estudio observacional transversal de indicación-prescripción.

Emplazamiento: Atención Primaria en la provincia de Orense.

Participantes: Todos los casos de EPOC incluidos en el registro de pacientes crónicos de 28 cupos de 9 Centros de Salud de la provincia de Orense.

Mediciones principales: Se determinó: edad, sexo, datos espirométricos, adecuación diagnóstica según resultados espirométricos y tratamiento farmacológico por vía inhalada (anticolinérgicos de larga duración, corticoides inhalados, beta-2 de larga duración y beta-2 de corta duración).

Resultados: Eran varones $297(77,7 \%)$ casos, con una media de edad de 77,0 (DE 11,0) años. El índice VEF1/CVF estaba registrado en 174 (45,5\%) pacientes, siendo menor de 0,7 en 138 casos $(36,1 \%)$, y figurando el VEF1 en 125 casos $(90,6 \%)$. Fueron clasificados como Graves o Muy graves 71 pacientes $(56,8 \%)$. El tratamiento era conforme a las recomendaciones en el $26,4 \%$ de los casos. En el $19,2 \%$ era incorrecto por defecto y en el $54,4 \%$ por exceso de medicación, relacionado fundamentalmente con el uso de corticoides inhalados, que estaban correctamente indicados en el $9 \%$ de los casos. No existían diferencias en el uso de fármacos en asociación en función de la gravedad de la EPOC.

Conclusiones: En la terapia de la EPOC con fármacos inhalados existe una baja adecuación a los criterios recomendados en las principales guías de práctica clínica, sobre todo en el uso de corticoides.

PALABRAS CLAVE: Enfermedad Pulmonar Obstructiva Crónica/Terapia. Broncodilatadores. Glucocorticoides. Adhesión a Directriz. Calidad de la Atención de Salud. Atención Primaria de Salud.

\section{ABSTRACT}

Objective: To assess the compliance of inhaled therapy in patients diagnosed with chronic obstructive lung disease to clinical guidelines (GOLD)

Design: Indication/prescription cross-sectional observational study

Location: Primary care in the province of Orense

Participants: All cases of COPD included in the register for 28 chronic patients in 9 health centres in the province of Orense.

Main Surveyed Data. The following were determined: age, sex, spirometric data, adjustment of diagnosis based on spirometric results, and inhaled drug therapy (long-acting anticholinergics, inhaled corticosteroids, long-acting beta-2 and short-acting beta-2)

Results: 297 cases (77.7\%) were male, with an average age of 77.0 years. The FEV1/ FVC ratio was registered in $174(45.5 \%)$ patients. It was lower than 0.7 in 138 cases $(36.1 \%)$ and FEV1 appeared in 125 cases (90.6\%). 71 patients $(56.8 \%)$ were classified as severe or very severe. The treatment was in accordance with recommendations in $26,4 \%$ of the cases. In $19.2 \%$ it was incorrect by default and in $54.4 \%$, through over-medication, related primarily to the use of inhaled corticosteroids, which were correctly indicated in $9 \%$ of cases. There were no differences in the use of associated drugs based on the severity of the COPD.

Conclusions: In COPD therapy with inhaled drugs, there is a low level of compliance with criteria recommended in the main clinical practice guidelines, especially regarding the use of corticosteroids.

KEYWORDS: Chronic obstructive pulmonary disease/therapy. Bronchodilators. Glucocorticoids. Adhesion to guidelines. Quality of healthcare. Primary healthcare. 


\section{INTRODUCCIÓN}

La Enfermedad Pulmonar Obstructiva Crónica (EPOC) es una enfermedad prevenible y tratable con algunos efectos extra-pulmonares significativos que pueden contribuir a la gravedad en pacientes individuales. Su componente pulmonar se caracteriza por limitación al flujo aéreo que no es totalmente reversible. Esta limitación habitualmente es progresiva y se asocia a una respuesta inflamatoria anormal del pulmón a las partículas nocivas o gases perjudiciales (humo de tabaco) ${ }^{1}$.

La EPOC es un problema socio-sanitario de primera magnitud por su elevada prevalencia, el constante aumento de su incidencia, la importante morbimortalidad que conlleva y el elevado consumo de recursos sanitarios que ocasiona ${ }^{2}$.

Diferentes recomendaciones, como las de la guía GOLD $^{1}$ o las de la ATS/ERS ${ }^{3}$ han subrayado la importancia de la detección precoz y sugieren que debería considerarse en cualquier paciente con síntomas de disnea, tos crónica o producción de esputo, o una historia de exposición a factores de riego de la enfermedad. El diagnóstico se confirma mediante espirometría postbroncodilatación (EF).

Ambas recomendaciones ${ }^{1,3}$ indican que el manejo de la EPOC estable debe incluir la determinación de la gravedad de la enfermedad, teniendo en cuenta los síntomas, la limitación al flujo aéreo, la frecuencia y severidad de las exacerbaciones, la insuficiencia respiratoria, la comorbilidad y el estado general de salud del paciente, estipulaciones que se han concretado en una modificación de la última revisión de la guía GOLD ${ }^{4}$.

El estudio IBERPOC estimó que la prevalencia de la EPOC en España era del 9,1\% con un amplio predominio en varones. En ese estudio, el 21,8\% se encontraba previamente diagnosticado y $19,3 \%$ de los pacientes estaban tratados. Así mismo, menos de la mitad de los pacientes identificados como EPOC grave (FEV1 $<50 \%$ ) recibían algún tipo de tratamiento para su enfermedad respiratoria. Esta situación se repite en otros estudios epidemiológicos en España ${ }^{5,6}$.

En cuanto a la adecuación a las guías clínicas (GPC) del tratamiento de los pacientes diagnosticados de EPOC, en diversos estudios se ha evidenciado que no siempre se corresponde con la terapéutica recomendada, apreciándose un alto uso de corticoides inhalados, teofilinas y mucolíticos, y un menor uso de anticolinérgicos, datos que no se corresponden con las recomendaciones vigentes ${ }^{7-10}$.

Con estas consideraciones, el objetivo del presente estudio fue evaluar la adecuación del tratamiento inhalado de los pacientes diagnosticados de EPOC a las recomendaciones vigentes en la guía GOLD 2006 (actualización GOLD 2010) ${ }^{1}$ en el momento de su realización.

\section{SUJETOS Y MÉTODOS}

Se realizó un estudio multicéntrico, transversal, de indicación-prescripción, en el ámbito de la Atención Primaria. Para ello, se seleccionaron la totalidad de los casos que con el diagnóstico de EPOC figuraban incluidos en el registro de crónicos (criterio único de inclusión) de 28 cupos de Medicina de Familia pertenecientes a 9 Centros de Salud, rurales y urbanos, de la provincia de Orense. La selección de los centros se realizó a partir de criterios de accesibilidad, pudiendo considerarse un muestreo de conveniencia. No se prejuzgó el estilo de práctica del profesional seleccionado y se solicitó su consentimiento para proceder a la recogida de datos. Ésta se realizó en forma de auditorías de la información contenida en las historias clínicas, por parte de personal médico, mediante procedimiento previamente acordado y a través de un formulario en el que se registraron los datos utilizados en el estudio. Los datos se recogieron, respetando el anonimato tanto del paciente como del profesional médico, entre los meses de noviembre de 2009 y junio de 2010. Se solicitó y obtuvo la autorización del Comité Ético de Investigación Clínica de Galicia.

Se determinó el consumo de fármacos inhalados para el tratamiento de la EPOC, en concreto corticoides inhalados $(\mathrm{CINH})$, agonistas $\mathrm{B2}$ de acción prolongada (B2L), agonistas B2 de acción corta (B2C) y anticolinérgicos de acción prolongada (ACL). Previamente, para una publicación anterior ${ }^{11}$, en las mismas historias clínicas, se habían recogidoy analizado los datos relacionados con el diagnóstico de la EPOC. También se comprobó el número de exacerbaciones (entendidas como aumento agudo de disnea, tos o esputo basal del paciente, más allá de las variaciones diarias ${ }^{1}$ ) en los 12 meses previos a la fecha de recogida de la información. El resto de las variables registradas fueron edad, sexo, valores obtenidos en la espirometría y adecuación diagnóstica a los criterios espirométricos según la guía GOLD utilizada como referencia'. El tratamiento se consideró adecuado si se ajustaba a lo indicado en la guía en función del estadiaje por gravedad según FEV1 posbroncodilatador y número de exacerbaciones.

El análisis de los datos se realizó con ayuda del programa SPSS versión 15.0 (SPSS Inc.). Las variables cuantitativas se describieron como media y desviación estándar (DE) y las cualitativas como porcentajes. Se determinaron los intervalos de 
confianza del 95\% (IC95\%). Se utilizó en el análisis la prueba t de Student de comparación de medias, $\chi^{2}$ y correlación lineal según fue necesario. El grado de significación estadística que se consideró fue un valor de $p<0,05$.

\section{RESULTADOS}

Fueron incluidos 382 casos, de los que 297 (77,7\%) eran varones, con una media de edad de 77,0 (DE $11,0)$ años. Se encontraban bien diagnosticados 138 casos $(36,1 \%)$, considerándose así aquellos en los que constaba en su historia clínica un índice VEF1/CVF $<0,7$ obtenido en una espirometría con prueba broncodilatadora. En 244 casos no había datos en la historia clínica que permitieran sustentar la existencia de un diagnóstico correcto.

En 125 de los 138 casos bien diagnosticados figuraba registrado el VEF1 y, por tanto, se podía clasificar su gravedad, de acuerdo con los criterios de la guía GOLD utilizada como referencia ${ }^{1}$. Con ese criterio fueron clasificados como enfermedad Grave/Muy grave $71(56,8 \%)$ individuos y como Leve/Moderada $54(43,2 \%)$ casos. Los pacientes con diagnóstico Grave/Muy grave eran seguidos más frecuentemente en atención especializada de segundo nivel $(70,4 \%)$. Los pacientes clasificados como Grave/Muy grave presentaban un mayor número de exacerbaciones que los clasificados como Leve/Moderado (1,9 frente a 1,0; IC95\% de la diferencia de medias $0,4-1,4 ; p=0,001$ ), no presentándose una fuerte asociación lineal negativa, aunque significativa, entre el valor del VEF1 y el número de exacerbaciones (Rho de Spearman -0,3; $p=0,002$ ).

La tabla 1 muestra la utilización de los diferentes fármacos en función del cumplimiento del criterio diagnóstico de la EPOC en el total de la muestra. La tabla 2 muestra la frecuencia de uso de algunos

\begin{tabular}{|c|c|c|c|c|}
\hline Fármaco & $\begin{array}{l}\text { Cumple criterio diagnóstico } \\
\qquad(n=138)\end{array}$ & $\begin{array}{l}\text { No cumple criterio } \\
\text { diagnóstico } \\
(n=244)\end{array}$ & $\chi^{2}$ & $\mathbf{p}$ \\
\hline B2C & $52(37,7 \%)$ & $62(25,4 \%)$ & 6,3 & 0,01 \\
\hline B2L & $104(75,4 \%)$ & $150(61,5 \%)$ & 7,6 & $<0,01$ \\
\hline$A C L$ & $79(57,2 \%)$ & $76(31,1 \%)$ & 24,9 & $<0,001$ \\
\hline $\mathrm{ClHN}$ & $106(76,8 \%)$ & $162(66,4 \%)$ & 4,6 & 0,03 \\
\hline
\end{tabular}

Tabla 1. Relación entre uso de los diferentes fármacos y presencia de criterio diagnóstico de la EPOC en la historia clínica en el total de la muestra.

B2C: agonistas $\beta 2$ de acción corta. B2L: agonistas $\beta 2$ de acción prolongada. ACL: anticolinérgicos de

\begin{tabular}{lcccccccccccc}
\hline \multirow{2}{*}{$\begin{array}{c}\text { Gravedad de la } \\
\text { enfermedad }\end{array}$} & \multicolumn{3}{c}{ CINH } & \multicolumn{3}{c}{ B2C } & \multicolumn{3}{c}{ B2L } & & \multicolumn{2}{c}{ ACL } \\
\cline { 2 - 12 } & Sí & No & $\chi^{2}(\mathbf{p})$ & Sí & No & $\chi^{2}(\mathbf{p})$ & Sí & No & $\chi^{2}(\mathbf{p})$ & Sí & No & $\chi^{2}(\mathbf{p})$ \\
\hline Grave/Muy grave & 56 & 15 & & 29 & 42 & & 57 & 14 & & 50 & 21 & \\
Leve/Moderada & 39 & 15 & $0,7(0,4)$ & 20 & 34 & $0,2(0,7)$ & 39 & 15 & $1,1(0,3)$ & 26 & 28 & $0,4(0,01)$ \\
Total & 95 & 30 & & 49 & 76 & & 96 & 29 & & 76 & 49 & \\
\hline
\end{tabular}

Tabla 2. Frecuencia de uso de los diferentes fármacos considerados en función de la gravedad de la EPOC clasificada por el valor del VEF1.

CINH: corticoides inhalados. B2C: agonistas $\beta 2$ de acción corta. B2L: agonistas $\beta 2$ de acción prolongada. ACL: anticolinérgicos de acción prolongada. 


\begin{tabular}{cccccc}
\hline Fármaco & \multicolumn{2}{c}{ Utilización } & Media de exacerbaciones (DE) & $\begin{array}{c}\text { IC95\% diferencia } \\
\text { de medias }\end{array}$ & p \\
\hline \multirow{2}{*}{ B2L } & Sí & 243 & $1,4(1,4)$ & $0,1-0,8$ & 0,001 \\
& No & 118 & $1,0(1,2)$ & & \\
\multirow{2}{*}{ ACL } & Sí & 149 & $1,6(1,6)$ & $0,3-0,9$ & $<0,001$ \\
& No & 212 & $1,0(1,2)$ & & \\
CINH & Sí & 256 & $1,4(1,5)$ & $0,1-0,9$ & $<0,001$ \\
& No & 105 & $0,9(1,1)$ & & \\
\hline
\end{tabular}

Tabla 3. Frecuencia de exacerbaciones y utilización de fármacos.

B2L: agonistas $\beta 2$ de acción prolongada. ACL: anticolinérgicos de acción prolongada. CINH: corticoides inhalados.

\begin{tabular}{|c|c|c|c|c|c|}
\hline Gravedad de la EPOC & \multicolumn{2}{|c|}{ Inhaladores combinados } & Total & $\chi^{2}$ & $\mathbf{p}$ \\
\hline Grave / Muy grave & 54 & 17 & 71 & & \\
\hline Leve / Moderada & 38 & 16 & 54 & 0,5 & 0,48 \\
\hline Total & 92 & 33 & 125 & & \\
\hline
\end{tabular}

Tabla 4. Uso de combinaciones de corticoides y agonistas $\beta 2$ de acción prolongada inhalados en pacientes con criterios de EPOC en función de su gravedad.

de los fármacos considerados en función de la gravedad de la enfermedad.

En el conjunto de los pacientes en los que era posible concretar las necesidades de tratamiento, era conforme a las guías el $26,4 \%$ de los casos, siendo incorrecto por defecto en el $19,2 \%$ y por exceso de medicación en el $54,4 \%$ de los individuos, en este caso sobre todo a expensas del uso incorrecto de los $\mathrm{CINH}$. Las mayores discrepancias se encontraban en los pacientes clasificados como leves y moderados (VEF1 > $50 \%$ ), en los que el porcentaje de tratamientos incorrectos era de $90,7 \%$. No se demostraron diferencias significativas en la adecuación del tratamiento en función del seguimiento por atención primaria o especialistas de segundo nivel $(p=0,4)$.

Si se acepta que los $\mathrm{CINH}$ sólo deben usarse en pacientes graves o muy graves (VEF1 < 50\%) con dos o más exacerbaciones en el último año', entonces 31 pacientes $(8,1 \%$ de la muestra) deberían utilizarlos. De los pacientes en los que estaba indicado el uso de $\mathrm{CINH}$, en 7 casos (22,6\% de los pacientes que debían usarlos) no se utilizaba. Por tanto, los que estaban bien indicados eran el 9,0\% (24/268) del total de los pacientes que los usaban. Por el contrario, los $\mathrm{CINH}$ se utilizaban en el $91,0 \%$ de los casos (244/268) sin existir constancia del criterio de indicación. Por otra parte, los $\mathrm{CINH}$ se utilizaban sin asociación a B2L o ACL en 29 casos (10,8\%). Las exacerbaciones eran más frecuentes en los grupos de pacientes que utilizaban más medicación (tabla 3 ).

Latabla 4 muestralautilización debroncodilatadores combinados (corticoides y agonistas B2 de acción prolongada) en función de la gravedad de la EPOC en pacientes con criterio diagnóstico correcto, no existiendo diferencia significativa en el uso de las combinaciones $\left(\chi^{2}=0,5 ; p=0,5\right)$.

Si se consideran sólo los pacientes clasificados como EPOC con criterios diagnósticos adecuados, 
el porcentaje de pacientes que utilizan inhaladores combinados es mayor entre los pacientes seguidos por atención especializada de segundo nivel $\left(80,9 \%\right.$ frente a $\left.59,3 \% ; \chi^{2}=7,8 ; p=0,005\right)$.

\section{DISCUSIÓN}

Con arreglo a los resultados obtenidos se puede afirmar que en la utilización de los fármacos inhalados considerados para el tratamiento de la EPOC existe un importante desfase entre la práctica observada y las recomendaciones aceptadas en las guías de uso habitual.

En concreto, se pueden distinguir dos circunstancias: por una parte parece existir un uso inadecuado de algunos fármacos, teniendo en cuenta que en un $64 \%$ de los casos no fue posible identificar criterios que permitieran el diagnóstico, lo que dificulta la estimación de la prevalencia de la adecuación y la comparación de resultados. En estudios similares realizados con el propósito de conocer la utilización de fármacos, Bourbeau et al encontraron en Canadá un $44 \%$ de pacientes en los que no se había realizado espirometría a pesar de estar en tratamiento por EPOC ${ }^{10}$. En el Reino Unido, Jones et al. hallaron un $27 \%$ de diagnósticos incorrectos ${ }^{12}$. En España, de Miguel et al. observaron un $37 \%$ de pacientes clasificados como EPOC y en los que, o no constaba EF, - los resultados no cumplían los criterios de $\mathrm{EPOC}^{13}$. Sin embargo, aún sin existir constancia del cumplimiento de los criterios diagnósticos, el consumo farmacológico de los pacientes fue analizado, encontrando que más del $60 \%$ de esos pacientes tenían prescritos $\mathrm{CINH}$ o B2L. A pesar de que las diferencias de uso de los diversos fármacos fueron siempre significativamente superiores en el grupo de pacientes con criterios diagnósticos adecuados, se comprobó una elevada utilización en pacientes sin criterios diagnósticos constatables en su historia clínica, lo que hace pensar en una utilización poco apropiada basada exclusivamente en criterios sintomáticos.

Por otra parte, se aprecia una inadecuada utilización de fármacos en relación con la categoría de gravedad a la que se encuentran asignados los pacientes en función de su VEF1, generalmente por exceso de tratamiento, hecho igualmente comprobado en otros estudios ${ }^{6,14-16}$.

La utilización de corticoides inhalados parece especialmente cuestionable, con más del $90 \%$ del total de pacientes incluidos utilizándolos sin criterios claros, y administrándose en los grupos de pacientes leves y moderados sin justificación, ya que no existen pruebas de mejores resultados al respecto ${ }^{17}$, apuntándose por algunos autores la extrapolación del efecto beneficioso que sobre la sintomatología del asma presentan estos fármacos ${ }^{10}$. Este mismo resultado fue constatado en Francia ${ }^{18}$, donde más del $50 \%$ de los pacientes en estadios I y II utilizaban $\mathrm{CINH}$; Reino Unido ${ }^{12}$, donde un $60 \%$ de los pacientes recibían $\mathrm{CINH}$, estando indicados en sólo el 23\%; o en Canadá ${ }^{10}$, donde más del $60 \%$ con enfermedad leve recibían $\mathrm{CINH}$. En una amplísima cohorte de 44.000 casos, Fitch en EE.UU. encontró un 8\% y un $19 \%$ de uso de $\mathrm{CINH}$ en pacientes leves y moderados respectivamente ${ }^{19}$. Viejo-Bañuelos et al. en nuestro país encontraron alrededor de un $70 \%$ de pacientes en estadio moderado y leve utilizándolos ${ }^{20}$, y de Miguel alrededor del $50 \%$ en esa misma circunstancia ${ }^{13}$. Este hecho redunda en un importante incremento del gasto por paciente en aquellos donde se utilizan indebidamente ${ }^{21}$. Por otra parte, en alrededor del $10 \%$ se usaban sin asociarse a broncodilatadores, en contra de lo recomendado en las GPC $^{1}$ y las evidencias de que los $\mathrm{CINH}$ sólo producen reducción de exacerbaciones cuando se utilizan en pacientes graves o muy graves ${ }^{17}$, y el hecho de que su uso está asociado con un aumento del riesgo de neumonía ${ }^{22}$.

Además, y aunque en menor cuantía, existen también pacientes que no reciben tratamiento a pesar de la existencia de datos que lo harían recomendable. Takahashi et al. encontraron en Japón un resultado similar en un amplio estudio realizado tanto sobre pacientes hospitalarios como de Atención Primaria ${ }^{23}$, lo mismo que un reciente estudio llevado a cabo en EE.UU. ${ }^{24}$.

Como en revisiones previas ${ }^{25}$, sigue habiendo un importante campo de mejora en el manejo de la EPOC. Probablemente, la primera fase de cualquier avance en el ámbito de la prescripción debería pasar por una mejora del proceso diagnóstico de estos pacientes ${ }^{26,27}$, medida cuya efectividad podría ser objeto de estudios de investigación ulteriores.

Entre las limitaciones propias de este estudio de indicación-prescripción es necesario considerar el hecho de que los datos proceden de un registro y puede contener ausencia de información o inexactitudes. A la hora de valorar la adecuación terapéutica se parte de la consideración de que el registro diagnóstico es adecuado, lo que no es necesariamente cierto. Además estos estudios pueden detectar mejor la infrautilización en tratamientos crónicos y enfermedades frecuentes que la sobreutilización, dado que los enfermos no diagnosticados quedan fuera del estudio. Por otra parte, es preciso considerar también que la selección de los cupos no se hizo de 
forma aleatoria, sino en función de un criterio de accesibilidad, lo que limita la representatividad de la muestra. No obstante, dado que no hubo negativas a participar por parte de ningún profesional, puede considerarse que la selección representa de forma adecuada la práctica clínica habitual.

En conclusión, el tratamiento farmacológico de la EPOC se basa en el uso escalonado de broncodilatadores. Para llevar a cabo un tratamiento idóneo de la enfermedad es necesario ceñirse a las recomendaciones que hacen de la espirometría la prueba imprescindible para valorar su gravedad. Existe una elevada discordancia entre el tipo de tratamiento empleado y la gravedad de la enfermedad según los valores obtenidos en la espirometría. En general, se observa un importante exceso de tratamiento a expensas del uso de corticoesteroides inhalados.

\section{BIBLIOGRAFÍA}

1. Global Initiative for Chronic Obstructive Lung Disease. Global strategy for the diagnosis, management, and prevention of chronic obstructive pulmonary disease. Updated 2010 [consultado 11 abril 2011]. Disponible en: http://www.goldcopd.org/uploads/users/files/ GOLDReport_April112011.pdf.

2. De Miguel Díez J. Fármaco-economía en el asma y en la EPOC. Arch Bronconeumol. 2005; 41: 239-41.

3. Celli BR, MacNee W; ATS/ERS Task Force. Standards for the diagnosis and treatment of patients with COPD: a summary of the ATS/ERS position paper. Eur Resp J. 2004; 23: $932-46$.

4. Global Initiative for Chronic Obstructive Lung Disease. Global strategy for the diagnosis, management, and prevention of chronic obstructive pulmonary disease (Revised 2011) [consultado 10 enero 2012]. Disponible en http://www.goldcopd.org/uploads/users/files/GOLD_ Report_2011_Jan21.pdf

5. Peña VS, Miravitlles $M$, Gabriel $R$, Jiménez-Ruiz $C A$, Villasante C, Masa JF et al. Geographic variations in prevalence and underdiagnosis of COPD: results of the IBERPOC multicentre epidemiological study. Chest. 2000; 118: $981-9$

6. Sobradillo V, Miravitlles M, Jiménez CA, Gabriel R, Viejo JL, Masa JF et al. Epidemiological study of chronic obstructive pulmonary disease in Spain (IBERPOC): prevalence of chronic respiratory symptoms and airflow limitation. Arch Bronconeumol. 1999; 35: 159-66.

7. Foster JA, Yawn BP, Maziar A, Jenkins T, Rennard SI, Casebeer L. Enhancing COPD management in primary care settings. MedGenMed. 2007; 9: 24.

8. Yawn BP, Wollan PC. Knowledge and attitudes of family physicians coming to COPD continuing medical education. Int J Chron Obstruct Pulmon Dis. 2008; 3: 311-7.

9. Chavez PC, Shokar NK. Diagnosis and management of chronic obstructive pulmonary disease (COPD) in a primary care clinic. COPD. 2009; 6: 446-51.

10. Bourbeau J, Sebaldt RJ, Day A, Bouchard J, Kaplan A, Hernández $P$ et al. Practice patterns in the management of chronic obstructive pulmonary disease in primary practice: The CAGE study. Can Respir J. 2008; 15: 13-9.

11. Díaz Grávalos GJ, Palmeiro Fernández G, Valiño López MD, Robles Castiñeiras A, Fernández Silva MJ, Reinoso
Hermida S et al. Adecuación diagnóstica en la Enfermedad Pulmonar Obstructiva Crónica. Rev Calid Asist. 2012; 27: 305-10.

12. Jones RCM, Dikson-Spillmann $M$, Mather MJC, Marks D, Shackell BS. Accuracy of diagnostic registers and management of chronic obstructive pulmonary disease: the Devon primary care audit. Respir Res. 2008; 9: 62.

13. De Miguel Díez J, Izquierdo Alonso JL, Rodríguez GonzálezMoro JM, de Lucas Ramos P, Molina Paría J. Tratamiento farmacológico de la EPOC en dos niveles asistenciales. Grado de adecuación a las normativas recomendadas. Arch Bronconeumol. 2003; 39: 195-202.

14. Miravitlles M, Murio $C$, Tirado-Conde G, Levy G, Muellerova $\mathrm{H}$, Soriano JB. Geographic differences in clinical characteristics and management of COPD: the EPOC Study. Int J Chron Obstruct Pulmon Dis. 2008; 3: 803-14.

15. Riario-Sforza GG, Incorvaia $C$, Pravettoni $C$, Dugnani N. Guidelines versus clinical practice in the treatment of COPD: a reappraisal. Eur Respir J. 2006; 27: 656.

16. Franssen F, Spruit MA, Wouters EFM. Determinants of polypharmacy and compliance with GOLD guidelines in patients with chronic obstructive pulmonary disease. Int $\mathrm{J}$ Chron Obstruct Pulmon Dis. 2011; 6: 493-501.

17. Puhan MA, Bachmann LM, Kleijnen J, Ter Riet G, Kessels AG. Inhaled drugs to reduce exacerbations in patients with chronic obstructive pulmonary disease: a network metaanalysis. BMC Med. 2009; 7: 2.

18. Jebrak G, pour Initiatives BPCO. Recommandations et prise en charge de la BPCO en France: les recommandations sur la prise en charge de la BPCO ne sont pas suivis dans la vraie vie. Rev Mal Respir. 2010; 27: 11-8.

19. Fitch K, Iwasaki K, Pyenson B, Plauschinat C, Zhang J. Variation in adherence with Global Initiative for Chronic Obstructive Lung Disease (GOLD) drug therapy guidelines: a retrospective actuarial claims data analysis. Curr Med Res Opin. 2011; 27: 1425-9.

20. Viejo-Bañuelos JL, Puedo-Bastida A, Fueyo-Rodríguez A. Characteristics of outpatients with COPD in daily practice: The E4 Spanish project. Respir Med. 2006; 100: 2137-43.

21. De Miguel-Díez J, Carrasco-Garrido P, Rejas-Gutiérrez J, Martín-Centeno A, Gobartt-Vázquez E, Hernández-Barrera $\checkmark$ et al. Inappropriate overuse of inhaled corticosteroids for COPD patients: impact on health costs and health status. Lung. 2011; 189: 199-206.

22. Calverley PM, Stockley RA, Seemungal TA, Hagan G, Willits LR, Riley JH et al; Investigating New Standards for Prophylaxis in Reduction of Exacerbations (INSPIRE) Investigators. Reported pneumonia in patients with COPD: findings from the INSPIRE study. Chest. 2011; 139: 505-12.

23. Takahashi $T$, Ichinose $M$, Inoue $H$, Shirato $K$, Hattori $T$, Takishima T. Underdiagnosis and undertreatment of COPD in primary care settings. Respirology. 2003; 8: 504-8.

24. Make B, Dutro MP, Paulose-Ram R, Marton JP, Mapel DW. Undertreatment of COPD: a retrospective analysis of US managed care and Medicare patients. Int $\mathrm{J}$ Chron Obstruct Pulmon Dis. 2012; 7: 1-9.

25. Izquierdo Alonso JL, Rodríguez González-Moro JM, de Lucas Ramos P, Martín Centeno A, Gobartt Vázquez E. ¿Ha cambiado el manejo de la EPOC en España? Resultados de un estudio multicéntrico comunitario (VICE). Rev Clin Esp. 2008; 208: 18-25.

26. Buffels J, Degryse J, Liistro G. Diagnostic certainty, comorbidity and medication in a primary care population with presumed airway obstruction: the DIDASCO2 study. Prim Care Respir J. 2009; 18: 34-40.

27. Lucas AE, Smeenk FW, Smeele IJ, van Schayck CP. Overtreatment with inhaled corticosteroids and diagnostic problems in primary care patients, an exploratory study. Fam Pract. 2008; 25: 86-91. 